\title{
Ustekinumab Therapeutic Drug Monitoring - Impact on Clinical Practice: A Multicenter Cross-Sectional Observational Trial
}

\author{
Waqqas Afif ${ }^{1} \cdot$ Bernie Sattin $^{2} \cdot$ Dorota Dajnowiec $^{2,7} \cdot$ Reena Khanna $^{3} \cdot$ Cynthia H. Seow ${ }^{4}$ Martin Williamson ${ }^{2}$. \\ Kinda Karra ${ }^{2,8} \cdot$ Yanli Wang ${ }^{5} \cdot$ Long-long Gao ${ }^{5} \cdot$ Brian Bressler $^{6}(1)$
}

Received: 30 March 2021 / Accepted: 9 July 2021 / Published online: 17 August 2021

(c) The Author(s) 2021

\begin{abstract}
Background and Aims The value of ustekinumab (UST) therapeutic drug monitoring (TDM) in clinical practice remains unclear. This study examined the impact of UST TDM on clinical decision making in patients with Crohn's disease (CD). Methods A total of 110 consecutive UST-treated CD patients were enrolled in this multicenter, single-arm cross-sectional study. During a single study visit, clinical decisions, disease characteristics, and serum and fecal samples were obtained. The primary outcome was congruency of the actual and two hypothetical clinical decisions based on provision of UST TDM (with and without fecal calprotectin [FCP]) to participating clinicians. Decisions were compared against those of a review panel. A sub-study retrospectively measured the associations of clinical outcomes at the next follow-up visit with serum UST concentration [UST].

Results No differences in the pattern of decisions by clinicians were observed before and after provision of UST TDM $(P=1.0)$ or UST TDM + FCP $(P=0.86)$. However, $39 \%(\mathrm{TDM})$ and 50\% (TDM + FCP) of hypothetical decisions differed from the initial decisions. The review panel's decisions differed with the addition of TDM +FCP $(P=0.0006)$, but not TDM alone $(P=0.16)$. The sub-study $(n=53)$ failed to detect an association between therapeutic serum [UST] at the initial study visit and clinical outcomes at the next visit.

Conclusions In consecutive CD patients treated with UST, the addition of TDM into routine clinical practice did not significantly impact clinical decisions and there was no association between short-term clinical outcomes and serum [UST]. Further studies are warranted before clinicians routinely implement UST TDM into clinical practice.
\end{abstract}

Keywords Treatment decision making $\cdot$ Treatment optimization $\cdot$ Serum concentrations $\cdot$ Anti-drug antibodies to ustekinumab

\section{Abbreviations}

$\mathrm{ADAb} \quad$ Anti-drug antibodies to ustekinumab

AE Adverse event

Anti-TNF Anti-tumor necrosis factor alpha antagonist

CD Crohn's disease

CI Confidence interval

Brian Bressler

brian_bressler@hotmail.com

1 Division of Gastroenterology and Hepatology, Department of Medicine, McGill University, Montreal, QC, Canada

2 Medical Affairs, Janssen Inc, Toronto, ON, Canada

3 Division of Gastroenterology, University of Western Ontario, London, ON, Canada

4 Division of Gastroenterology and Hepatology, University of Calgary, Calgary, AB, Canada
ELISA Enzyme-linked immunosorbent assay

FCP Fecal calprotectin

HBI Harvey-Bradshaw index

IBD Inflammatory bowel disease

IFX Infliximab

IQ Interquartile
5 Janssen R\&D, Spring House, PA, USA

6 Division of Gastroenterology, Department of Medicine, Faculty of Medicine, University of British Columbia, 5th Floor, 2775 Laurel Street, Vancouver, BC V5Z 1M9, Canada

7 Present Address: Edwards Lifesciences Corp., One Edwards Way, Irvine, CA 92614, USA

8 Present Address: Merck Canada Inc., 16750 Trans-Canada Hwy, Kirkland, QC H9H 4M7, USA 


$\begin{array}{ll}\text { IQR } & \text { Interquartile range } \\ \text { IRB } & \text { Institutional review board } \\ \text { IV } & \text { Intravenous } \\ \text { OR } & \text { Odds ratio } \\ \text { PK } & \text { Pharmacokinetic } \\ \text { RIA } & \text { Radioimmunoassay } \\ \text { SC } & \text { Subcutaneous } \\ \text { SD } & \text { Standard deviation } \\ \text { UC } & \text { Ulcerative colitis } \\ \text { UST } & \text { Ustekinumab }\end{array}$

\section{Introduction}

Therapeutic drug monitoring (TDM) is an important part of the management of inflammatory bowel disease (IBD) $[1,2]$. It has been demonstrated to be cost-effective and associated with improved therapeutic outcomes compared to empiric treatment [3, 4]. TDM is commonly used to guide clinical decisions related to dose optimization at the time of loss of response to tumor necrosis factor alpha antagonists (antiTNFs) in patients with Crohn's disease (CD) [5]. Therapeutic trough concentration assessments have been suggested for patients with secondary failure to anti-TNF medications [1, $3,4]$, and concentration-based dose optimization has been shown to guide clinical decisions that result in increased remission rates and reduced hospitalizations in patients with active IBD $[4,6,7]$. The addition of fecal calprotectin (FCP) testing to anti-TNF TDM may further optimize management decisions in IBD [8]. The potential utility of TDM for predicting future response to therapy and for proactively adjusting anti-TNF dosage to prevent loss of response has also been suggested $[9,10]$. There is less evidence to support the use of TDM and FCP for other classes of biologics, including ustekinumab (UST), in the clinical setting.

Ustekinumab has been demonstrated effective for the treatment of CD [11] and ulcerative colitis (UC) [12], and there is a growing body of evidence relating to exposure-efficacy relationships [13-17] although there remains a lack of consensus on serum [UST] thresholds at different time points [13]. Exposure-efficacy data suggest that a drug concentration of $>1 \mu \mathrm{g} / \mathrm{ml}$ is associated with improved clinical and endoscopic outcomes [13]. Different assays have been used in studies of UST TDM to date adding further ambiguity [18, 19], and the real-world clinical utility of UST TDM on clinical decision making remains poorly described in the literature relative to what is known about TDM for anti-TNFs.

We conducted a cross-sectional congruency study to address these gaps in the understanding of UST TDM. The primary objective was to evaluate the impact of providing TDM-related information (i.e., serum [UST] and anti-drug antibodies [ADAb] to ustekinumab) with and without information from FCP testing, on clinical decisions in patients with CD in a real-world clinical setting. We hypothesized that the provision of UST TDM with or without FCP results would impact clinical decisions made in the management of CD patients treated with UST and therefore establish a role for TDM in the treatment of CD patients with UST. We explored the association between UST TDM \pm FCP-related information and measures of CD activity. A sub-study retrospectively examined the association between UST TDM results (i.e., "therapeutic" versus "subtherapeutic" serum [UST]) and treatment outcomes at the next follow-up visit. Taken together, these three critical pieces of information could help rationalize the incorporation of UST TDM into clinical practice.

\section{Methods}

\section{Study Population}

Consecutive outpatients aged 18 to 80 years with documented CD who had been initiated on UST either subcutaneously (SC) or intravenously (IV) for at least 4 weeks with the most recent dose of UST within the last 12 weeks were eligible. Patients were excluded if they had a confirmed diagnosis of UC, an ostomy, or prior extensive bowel resection.

\section{Study Design}

This was a cross-sectional, multicenter, non-interventional study conducted in 11 Canadian sites that had experience using TDM as a decision-making tool for patients on antiTNFs. Study enrollment occurred between April 2017 and January 2018. The institutional review board at each study site approved the protocol (see Supplement for additional information on protocol amendments), and all patients provided written informed consent. During the single study visit, patients provided a medical and medication history and completed the Harvey-Bradshaw index (HBI) questionnaire for disease activity (Fig. 1). A blood sample was obtained for TDM (i.e., serum [UST] and [ADAb] to ustekinumab); this was not protocolized therefore not necessarily at trough. The treatment decision (D1) was taken prior to, and independent of, the patient's inclusion in the study and according to standard clinical practice. UST TDM results were provided to participating clinicians after their decisions were made, and they recorded whether their treatment decision would hypothetically change based on the provision of UST TDM results alone (D2), and where available, TDM + FCP (D3).

A review panel of four expert gastroenterologists was convened, and each patient case was reviewed by three panel members who made a hypothetical clinical decision (D1) 


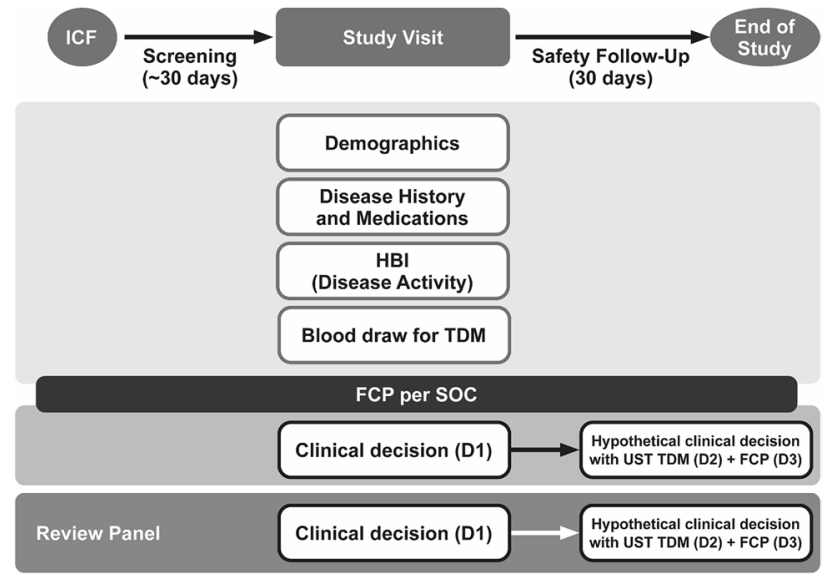

Fig. 1 mUST-DECIDE overall study design. FCP, fecal calprotectin; ICF, informed consent form; HBI, Harvey-Bradshaw index; SOC, standard of care; TDM, therapeutic drug monitoring; UST, ustekinumab

and then re-evaluated the case based on the provision of UST TDM (D2) and then UST TDM + FCP (D3).

\section{Study Evaluations}

The primary outcome was the congruency of clinical decisions made by participating clinicians before and after access to UST TDM results. Clinical decisions after access to TDM results included "no action" (i.e., no change in decision compared to baseline) or "action" (i.e., a change in decision compared to baseline, which could include request for further investigation such as laboratories, imaging [or other], dose optimization, treatment discontinuation, and treatment switch). Secondary outcomes included the congruency of additional decision pairs, including those of the review panel (i.e., D1 vs. D3 and D2 vs. D3). The review panel followed a majority rule approach for clinical decisions, which were not protocolized; if a consensus of at least two of three members was not reached, the case was labeled "disagreement."

Adverse events (AEs) were recorded from the time a signed and dated informed consent form was obtained until 30 days after the initial study visit.

\section{UST and ADAb to UST Serum Concentration Measurements}

Serum [UST] and presence of ADAb to UST were assessed using an enzyme-linked immunosorbent assay (ELISA) [20] (detection range $0.005-20 \mu \mathrm{g} / \mathrm{mL}$ ) and a drug-tolerant radioimmunoassay (RIA) [21] (lower limit of detection: 3 AU/mL), respectively (Sanquin Research Labs, Netherlands). A high level of agreement has been shown between the Sanquin UST and ADAb assay and the assay used in the UST registration trials [18] (and unpublished data from Sanquin).

\section{Sub-study}

A retrospective chart review was completed in patients who had a follow-up visit $\geq 30$ days after the initial visit. Improved disease control [22] (exploratory outcome) was defined as a composite assessment outcome meeting $\geq 1$ disease control criterion (symptomatic, endoscopic/imaging, biochemical) without any of the non-response criteria (inadequate or loss of response, worsening of any disease control criteria, initiation of any CD-related medications, and AEs). Serum [UST] was categorized as therapeutic, subtherapeutic, or uninterpretable based on their position in a two-compartment pharmacokinetic (PK) model. (Subjects on Q8W dosing were assessed as per the log-linear model which projected a therapeutic level of $\geq 4.5 \mu \mathrm{g} / \mathrm{ml}$ at 4 weeks and $\geq 1.0 \mu \mathrm{g} / \mathrm{ml}$ at 8 weeks.) This allowed for interpretation of non-trough sampling [13, 23].

\section{Statistical Methods}

A sample size of 100 patients was estimated to have approximately $90 \%$ power to detect a $15 \%$ change in clinical decisions (assuming 25\% discordant pairs). For the primary endpoint, a two-sided McNemar's test with a $P$ value of 0.05 was used. No corrections were made for multiple testing.

Statistical analyses were performed by or under the authority of the sponsor. All authors had access to the data and reviewed and approved the final manuscript.

\section{Results}

\section{Patient and Treatment Information}

A total of 110 patients were enrolled and completed the study. Patient demographics, baseline disease characteristics, and medication use are summarized in Table 1. The mean duration of UST therapy was 14.4 months (SD 12.5). Fifty-five patients received induction dosing with SC UST and 46 with IV UST. The median induction doses by body weight were $360 \mathrm{mg}$ and $390 \mathrm{mg}$ for SC and IV UST, respectively. During maintenance, 66 patients received UST therapy at 8-week intervals, with the remaining at 4-6-week intervals. The median disease duration was 16.2 years, the majority $(90.0 \%)$ had previously failed at least one antiTNF, the mean HBI score was $4.0( \pm 3.95)$, and $77(70.0 \%)$ patients were in remission (i.e., HBI score $<5$ ). 
Table 1 Demographics, baseline characteristics, and CD medication history $(n=110)$

\begin{tabular}{ll}
\hline Demographic characteristics & \\
Male, $n$ (\%) & $48(43.6)$ \\
White, $n(\%)$ & $100(90.9)$ \\
Age (yrs), mean (SD) & $43.2(13.67)$ \\
CD disease duration (yrs), median (range) & $16.2(1-42)$ \\
Baseline disease characteristics & \\
CD location, $n$ (\%) & \\
Ileum & $76(69.1)$ \\
Colon & $56(50.9)$ \\
Ileum and colon & $33(30.0)$ \\
Proximal small intestine, stomach, and/or esophagus & $10(9.1)$ \\
Perianal & $24(21.8)$ \\
Disease activity (HBI score) at initial study visit, $n$ & \\
(\%) & \\
Remission (HBI $<5)$ & $77(70.0)$ \\
Mild disease (HBI 5-7) & $14(12.7)$ \\
Moderate disease (HBI $8-16)$ & $18(16.4)$ \\
Severe disease (HBI $\geq 16)$ & $1(0.9)$ \\
Extra-intestinal manifestations, $n(\%)$ & $91(82.7)$ \\
Arthritis/arthralgia & $36(39.6)$ \\
Anal fissure, fistula, or abscess & $15(16.5)$ \\
Perianal fistulae & $5(5.5)$ \\
Rectovaginal fistulae & $5(5.5)$ \\
Abdominal fistulae & $1(1.1)$ \\
CD medication history & \\
Prior CD therapies, $n(\%)$ & \\
Anti-TNF & \\
Immunosuppressants & $99(90.0)$ \\
Corticosteroids & $87(79.1)$ \\
Current/concomitant CD therapies, $n(\%)$ & $82(74.5)$ \\
Immunosuppressants & $91(82.7)$ \\
Other biologics (vedolizumab) or investigational & \\
$\quad$ & \\
\hline
\end{tabular}

* Other agents included Bifidobacterium infantis, cannabis sativa, cholestyramine, folic acid, folinic acid, hydroxychloroquine sulfate, ketamine, Lactobacillus acidophilus, loperamide, loperamide hydrochloride, pentoxifylline, probiotic not otherwise specified, quercetin, VSL\#3

\section{Fecal Calprotectin}

Each patient was scheduled to have a FCP test per routine care, though FCP values were available for $72(65.5 \%)$ patients at the time of evaluation. The median (IQR) FCP was 208 (103-432) ug/uL. Among these 72 patients, the majority $(\mathrm{n}=51,70.8 \%)$ were in symptomatic remission, while $8(11.1 \%)$ had mild disease and $13(18.1 \%)$ had moderate disease, according to HBI scores.

\section{C-Reactive Protein (CRP)}

CRP results were available for $89(80.9 \%)$ patients with a median (IQR) of $3.3 \mathrm{mg} / \mathrm{L}$ (1.3-6.7), which included 63 (70.8\%) in HBI symptomatic remission, 10 (11.2\%) having mild disease, and 16 (18.0\%) having moderate disease.

\section{Primary Analysis: Clinical Decisions by Participating Clinicians Before and After TDM ( \pm FCP)}

Overall, treatment decisions by participating clinicians before (D1) and after the provision of UST TDM results (D2) were unchanged (Fig. 2a) (i.e., the number of actions changing to no further actions canceled out the number of no actions to actions). The most common "actions" (i.e., clinical decisions) were "dose optimization," followed by "treatment switch" and "further investigation" (Supplementary Table 1S). At an individual patient level, 39.1\% (95\% CI 29.8-48.4\%) of the hypothetical clinical decisions were different when UST TDM results were made available. The addition of FCP to TDM results (D3) also failed to have a net impact on treatment decisions (Fig. 2b, Supplementary Table 2S). At an individual level, $50.0 \%$ of decisions would have been different if UST TDM + FCP results had been available. The addition of FCP to UST TDM results did not change the net proportions of clinical decisions by participating clinicians (D3 versus D2, $P=0.10$; Supplementary Table $3 \mathrm{~S}$ ), and only $15.3 \%$ of individual decisions would have been different.

\section{Secondary Analysis: Clinical Decisions by Review Panel Before and After TDM ( \pm FCP)}

The review panel decisions were broadly similar to the primary clinical decisions, and the level of agreement across the review panel decisions was high (range: $83.3 \%$ to $95.5 \%$ ). The net proportions of clinical decisions were not different before (D1) and after the provision of UST TDM results (D2) (Fig. 3a), but they did change based on TDM +FCP results (D3) (Fig. 3b, Supplementary Table 4S). At an individual patient level, 22.7\% (95\% CI 14.8-30.7\%) of decisions would have been different if the panel had access to UST TDM results and $66.7 \%$ of individual decisions would have been different if UST TDM + FCP results had been available. The addition of FCP to UST TDM results changed the net proportions of clinical decisions by the expert panel (D3 versus D2, $P=0.004$; Supplementary Table $6 \mathrm{~S}$ ), and $59.7 \%$ of individual decisions would have been different. 
(a)

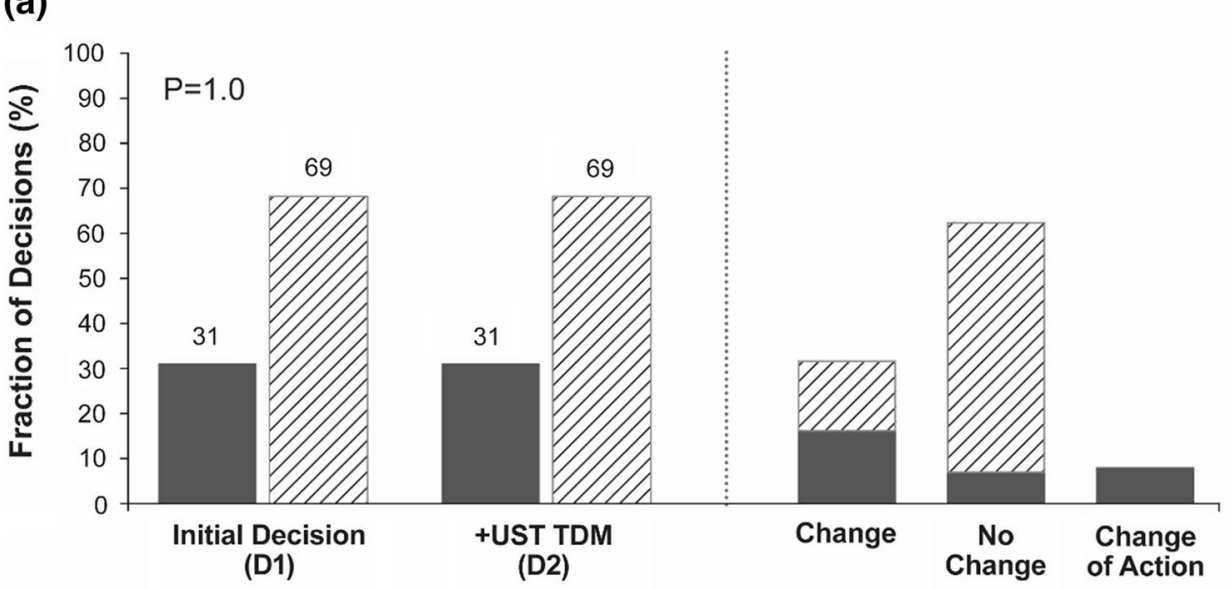

Action

No action

(b)

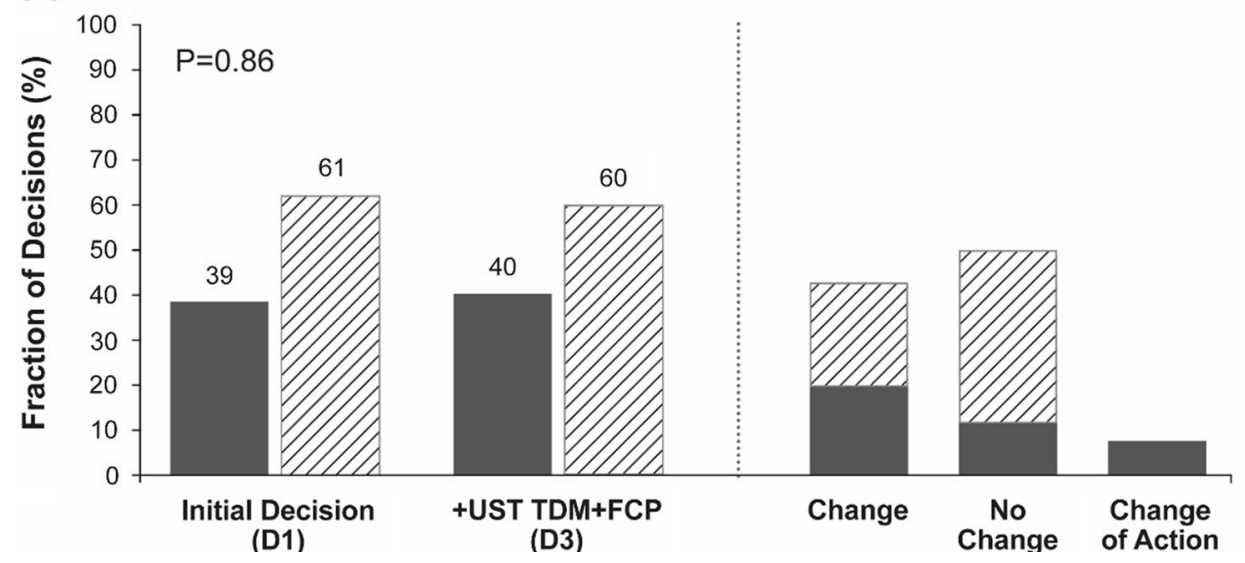

Fig. 2 Congruency of CD treatment decisions by participating clinicians before and after provision of a UST TDM ( $n=110$ ) and $\mathbf{b}$ UST TDM + FCP $(n=72)$ Results. FCP, fecal calprotectin; TDM, therapeutic drug monitoring; UST, ustekinumab

\section{Subgroup Analyses}

\section{Serum [UST] and [ADAb to UST]}

Sites were advised to take serum samples at trough; however, specific sampling was variable since this was not protocolized. Subgroup analyses of serum [UST] and [ADAb] were conducted only in patients who were sampled at trough. The median serum [UST] according to dose frequency and disease activity status is summarized in Table 2. Serum [UST] was generally higher in patients receiving $\mathrm{Q} 4 \mathrm{~W}$ dosing relative to Q8W dosing and appeared independent of disease activity status. No patient was positive for serum ADAb to UST.

\section{Sub-study}

A subset of 53 patients had a subsequent follow-up visit more than 30 days after the initial visit and an interpretable serum [UST]. Among them, $44(83.0 \%)$ had therapeutic, 9 (17.0\%) had subtherapeutic serum [UST], and $35(66.0 \%)$ were in symptomatic remission at the initial visit. In the subgroup of patients with available HBI at follow-up, five out of $17(29.4 \%)$ of the therapeutic subgroup had some clinical disease activity (HBI $\geq 5)$, whereas none $(n=0 / 4)$ of the subtherapeutic subgroup had disease activity (i.e., all patients had $\mathrm{HBI}<5$ ). Serum [UST] at baseline was not associated with clinical decisions (Fig. 4a). After a median of 148 days (range $41-411), 50.9 \%$ of patients $(n=27)$ were in complete disease control; their clinical outcomes appeared independent of achieving therapeutic serum [UST] at the initial visit (odds ratio [OR] $0.80,95 \%$ confidence interval [CI] 0.19-3.38; Fig. 4b).

\section{Safety}

Overall, 33 (30.0\%) patients had one or more AEs from enrollment until 30-day follow-up. The most common AEs 
(a)

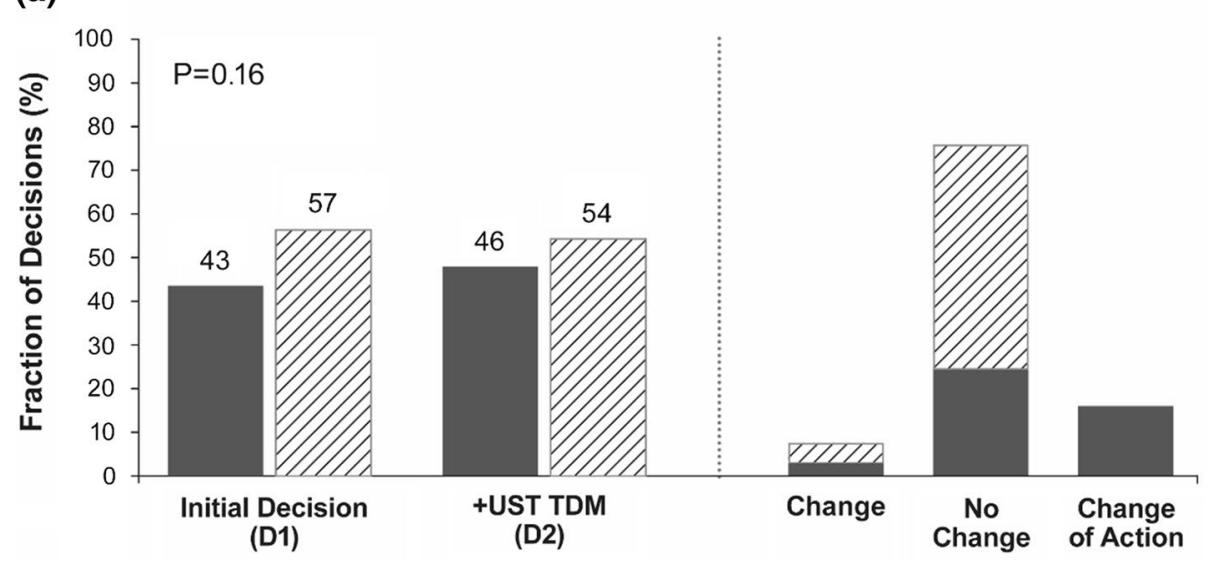

Action

W/. No action

(b)

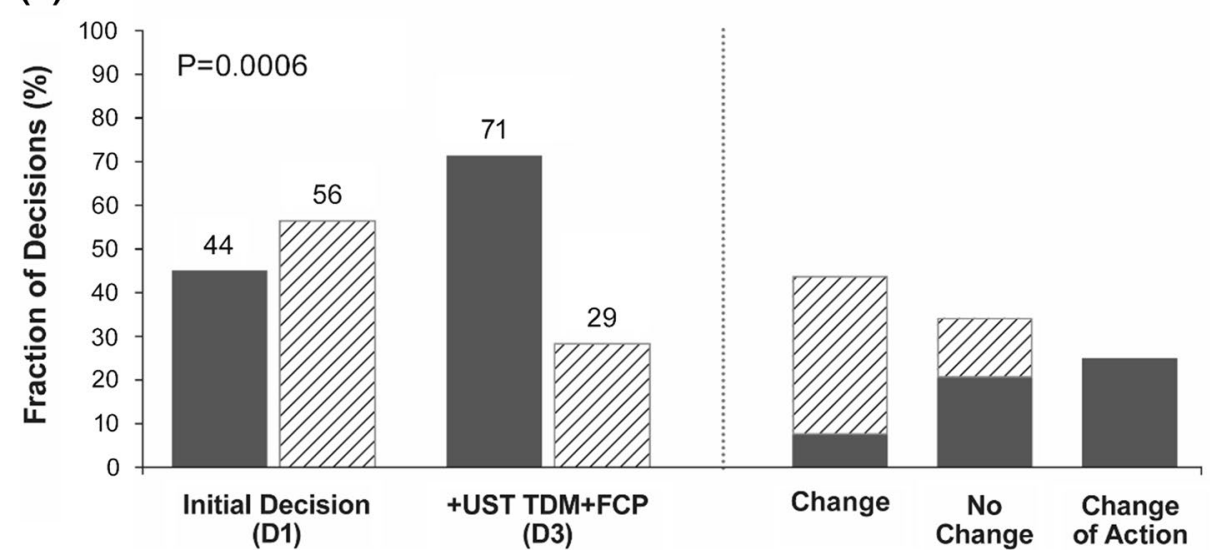

Fig. 3 Congruency of CD treatment decisions by the review panel before and after provision of a UST TDM $(n=110)$ and $\mathbf{b}$ UST TDM + FCP $(n=72)$ results. FCP, fecal calprotectin; TDM, therapeutic drug monitoring; UST, ustekinumab

Table 2 Median serum trough* [UST], $\mu \mathrm{g} / \mathrm{mL}$ (IQR; n), by dosing frequency and disease activity

\begin{tabular}{llllll}
\hline & \multicolumn{2}{l}{ Disease activity (HBI score) } & & \\
\cline { 2 - 6 } & Remission $(<5)$ & Mild $(5-7)$ & Moderate $(8-16)$ & Severe $(>16)$ & All patients \\
\hline Q8W & 3.9 & 2.3 & 3.6 & 0 & 3.6 \\
Median (IQR) & $(2.2-9.3 ; n=22)$ & $(0.9-2.7 ; n=3)$ & $(0.4-4.3 ; n=11)$ & & $(1.8-5.0 ; n=36)$ \\
Q4W & 9.9 & 11.0 & 4.0 & 0 & 9.7 \\
Median (IQR) & $(5.8-12.3 ; n=12)$ & $(9.3-12.2 ; n=4)$ & $(3.7-6.4 ; n=3)$ & & $(5.0-12.0 ; n=19)$ \\
\hline
\end{tabular}

*Trough was defined as the samples collected and measured when the patient visit date was \pm 7 days on next expected dose and at least 20 days after most recent injection, or visit date was $\leq 7$ days of most recent injection. All samples had UST injection of $90 \mathrm{mg}$ at the most recent prescribed dose

were drug inefficacy $(n=27,24.5 \%)$ (prior to the first protocol amendment, any subject with $\mathrm{HBI}>5$ was categorized as "lack of efficacy"), gastrointestinal disorders $(n=3,2.7 \%)$, and skin and subcutaneous tissue disorders $(n=2,1.8 \%)$. One patient reported one serious adverse event (small intestinal obstruction), which was judged by the investigator as unrelated to UST.

\section{Discussion}

mUST-DECIDE was a phase IV, cross-sectional, multicenter, non-interventional study conducted in patients with documented CD who were predominantly receiving UST maintenance therapy at baseline. Patients had 

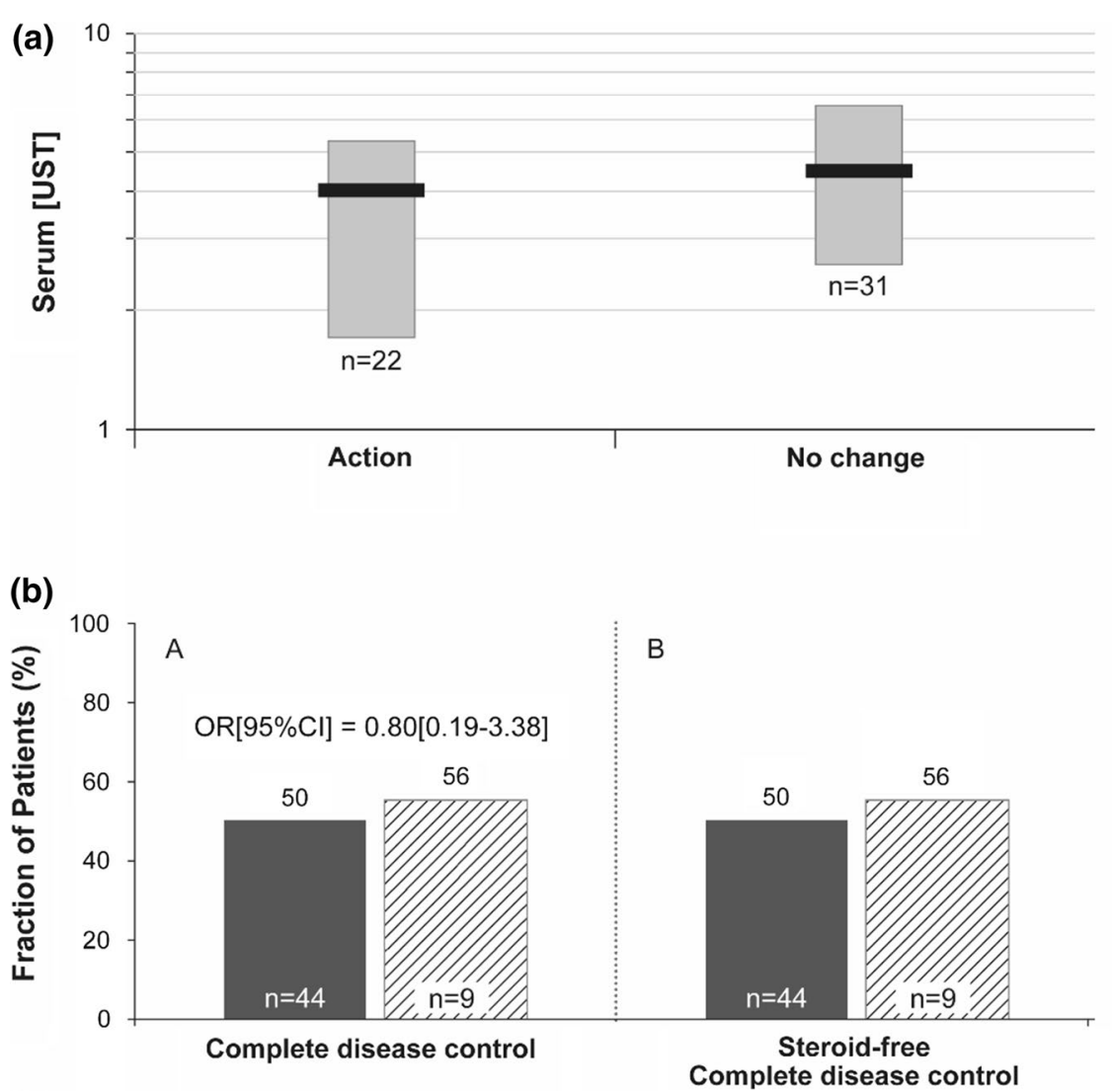

Fig. 4 a Association of serum [UST] with clinical decisions (action vs. no change) in the sub-study ( $n=53$ ) and $\mathbf{b}$ impact on disease outcomes at follow-up visit $(n=53)$. CI, confidence interval; OR, odds ratio; UST, ustekinumab

longstanding, highly refractory $\mathrm{CD}$, yet a majority were in remission.

The study failed to demonstrate an impact of routine UST TDM on clinical decisions. No effect was detected at the level of participating clinicians or a review panel consisting of gastroenterology experts. This contrasts with what has been reported with anti-TNFs for the treatment of CD [4, 6, 8, 24], where TDM is commonly used in clinical practice [1-3]. Despite the lack of a net difference in clinical decisions, the proportions of individual $\mathrm{CD}$ treatment decisions made by participating clinicians $(39.1 \%)$ and the review panel $(22.7 \%)$ changed after the provision of UST TDM results, indicating that the availability of TDM may impact clinical decision making. A proportion of these changed decisions $(23.6 \%$ in the expert group) were related to ordering further testing, presumably to assess for active disease (additional radiology testing/ FCP information). The changed clinical decisions tended to be uniform in both directions, with similar numbers of actions changing to no further actions, and vice versa, thus explaining the net neutral overall result. The statistical relevance of the changed decisions cannot be ascertained since there was no control group in this study, though they appear lower than expected from similar experiments with anti-TNFs $[4,6,8,24]$.

Similar studies have been reported in the anti-TNF literature. For example, a single-center study of 36 IBD patients from the University of Alberta found $69.4 \%$ of decisions would be different based on IFX TDM results [8].

Interpretation of UST TDM results remains poorly described in the literature compared to the widely accepted thresholds for anti-TNF TDM [1, 4, 7]. An analysis from the UNITI trials reported that serum [UST] was proportional to dose and treatment efficacy, which included clinical remission and endoscopic efficacy [13], and PK analyses suggested that trough concentration targets for clinical remission during maintenance treatment with UST ranged from 0.8 to $1.4 \mu \mathrm{g} / \mathrm{mL}$ [13]. Other cohort studies have suggested higher threshold UST maintenance levels in anti-TNF refractory CD patients ranging from 1.7 to $4.5 \mu \mathrm{g} / \mathrm{mL}[14,15$, 17]. In a Canadian cohort, highly refractory $C D$ patients were treated with UST SC during induction and optimized maintenance and showed improved clinical and endoscopic outcomes in patients with serum [UST] higher than $4.5 \mu \mathrm{g} /$ $\mathrm{mL}$ with a homogeneous mobility shift assay [14]. Notably, substantial absolute differences in [UST] have been reported 
between different assays [19], which may help explain the approximately twofold to threefold higher serum [UST] reported in these studies compared to the UNITI trials. Thus, there is no clear established threshold for therapeutic [UST] and median serum [UST] during maintenance treatment of CD has not been consistently shown to correlate with clinical or endoscopic remission rates [15, 17, 25]. In the mUST-DECIDE sub-study, a threshold of $1 \mu \mathrm{g} / \mathrm{ml}$ at trough was used to explore this association, given the PK data from the UNITI trials. In patients mainly in clinical remission, serum [UST] at the initial study visit did not predict clinical decisions (i.e., "action" or "no action") and there was no association between serum [UST] and short-term clinical outcomes at the next follow-up visit.

Access to FCP results after the UST TDM-related information did not alter decisions by participating clinicians but did change decisions by the review panel. The FCP information resulted in a larger proportion of changed decisions than with UST TDM alone (50\% and 66.7\% decisions by participating clinicians and the review panel, respectively). This larger proportion of changes in the review panel suggests a greater adherence to clinical guidelines to objectively measure active inflammation to inform clinical decisions [5]. Similar to the UST TDM results, changed decisions tended to balance out in both directions, with similar numbers of actions changing to no actions, and vice versa.

The proportions of changes to treatment decisions (based on UST TDM) after provision of FCP results were 15.3\% and $59.7 \%$ for decisions made by participating clinicians and the review panel, respectively. This suggests that in expert hands, FCP appears to influence CD treatment decisions and highlights the importance of a complete drug concentration and biomarker profile in properly assessing the clinical course of action, with the caveat that this study did not investigate the role of FCP alone. It remains possible that FCP alone could drive a majority of clinical decisions, suggesting a more limited role for UST TDM in the context of active inflammation. Fecal calprotectin has been demonstrated as a valuable monitoring $[26,27]$ and decision-making tool [8]. In fact, an algorithm has been proposed for IFX [28].

No patients in this study were positive for serum ADAb to UST, which is similar to other results varying from no ADAb [14-16] to very low incidence in the UNITI (2.3\%) [29] and UNIFI trials (4.6\%) [12]. Notably, the presence of ADAb in IM-UNITI did not preclude efficacy of UST and no definitive conclusions could be drawn on the effect of $\mathrm{ADAb}^{29}$.

This study has several limitations. Clinical management, including the UST dosing regimen, was not protocolized, in order to reflect the use of UST in the real world. A specific serum sampling time was also not protocolized nor uniformly executed, and thus, serum samples to determine [UST] were collected at trough in only $~ 50 \%$ of patients. This underscores the practical challenges of sampling UST-treated patients that are dosed subcutaneously every 8 to 12 weeks. Further, there are no published studies on interpretation of non-trough serum [UST], and it is unclear how the participating sites intended to interpret non-trough samples. Despite this, the review panel was able to extrapolate the therapeutic nature of the non-trough PK samples. FCP information was available for only a subset of 72 patients, and the sub-study included only 53 patients. The patient population comprised stable, treatment-resistant patients who had been exposed to multiple therapies and prior surgeries, possibly limiting the role of serum [UST] on clinical decision making-these patients had already exhausted their available treatment options and physicians may have been predisposed to maintaining their current dose in the absence of symptoms. The majority of patients were in remission (i.e., $\mathrm{HBI}<5$ ), and the findings should not be extrapolated to patients exhibiting a secondary loss of response to UST.

In conclusion, obtaining UST TDM information in the clinical management of consecutive $\mathrm{CD}$ patients on maintenance treatment with UST did not alter theoretical decision making for clinicians or an expert review panel, whereas adding FCP to UST TDM altered clinical decisions for the review panel but not clinicians. A sub-study showed no impact between baseline serum [UST] and short-term clinical outcomes at the next follow-up visit. The divergence on impact of TDM and FCP between clinicians and the review panel highlights a need for greater understanding and education in assessing for active inflammation using FCP and interpreting UST TDM results. Further studies to clarify the use and impact of these tests in clinical practice across different clinical scenarios (e.g., reactive/loss of response) are warranted.

Supplementary Information The online version contains supplementary material available at https://doi.org/10.1007/s10620-021-07173-1.

Author contributions WA, BS, DD, MW, KK, and BB were involved in study concept and design; DD, RK, CHS, and $\mathrm{BB}$ were involved in data acquisition; BS, DD, RK, CHS, MW, YW, LLG, and BB analyzed the data and were involved in interpretation; WA, BS, MW, and BB drafted the manuscript; WA, DD, RK, CHS, MW, YW, and LLG were involved in statistical analysis; BS and DD contributed to obtained funding; BS, DD, MW, KK, and WA supervised the study. All authors critically reviewed the manuscript for important intellectual content.

Writing assistance Writing support was provided by Christina Clark, Ardeane Healthcare Solutions, and funded by Janssen Inc. 
Funding This study was supported by Janssen Inc.

\section{Declarations}

Data availability statement Janssen's official data sharing statement ("The data sharing policy of Janssen Pharmaceutical Companies of Johnson \& Johnson is available at https://www.janssen.com/clinicaltrials/transparency. As noted on this site, requests for access to the study data can be submitted through Yale Open Data Access [YODA] Project site at http://yoda.yale.edu") will be provided to a journal/congress upon request.

Disclosure The authors disclose the following: WA: Advisor/Speaker for Janssen, AbbVie, Takeda, Arena Pharmaceuticals; Advisor to Pfizer, Merck, Ferring, Novartis, Amgen, Innomar; Research support from AbbVie, Prometheus, Theradiag, Eli-Lilly, Roche, Dynacare. BS, MW, YW, LLG: Employees of Janssen Inc. DD and KK: Former employees of Janssen Inc. RK: Advisory board member for AbbVie, Janssen, Takeda, Merck, Innomar, Lilly, Roche; Consultant to Robarts Clinical Trials, Encycle; Research support from Roche; Speaker for AbbVie, Janssen, Takeda, Lilly, Roche, Pfizer. CHS: Advisory boards/ Speaker for Janssen, AbbVie, Takeda, Ferring, Shire, Pfizer, PharmaScience. BB: Advisor/Speaker for Pfizer, Merck, Ferring, Janssen, AbbVie, Takeda, Celgene, Genentech; Advisor to Allergan, AMT; Research support from Janssen, AbbVie, Takeda, Atlantic Pharmaceuticals, GSK, BMS, Amgen, Genentech, Merck, RedHill Biopharm, BI, Qu Biologic, Celgene, Alvine. Stock options: Qu Biologic.

Ethics approval The study protocol and amendments were approved by an independent ethics committee or institutional review board. The trial was conducted in compliance with the ethical principles of the Declaration of Helsinki, Good Clinical Practices, and applicable regulatory requirements. All patients provided written informed consent before they participated in the study.

Open Access This article is licensed under a Creative Commons Attribution-NonCommercial 4.0 International License, which permits any non-commercial use, sharing, adaptation, distribution and reproduction in any medium or format, as long as you give appropriate credit to the original author(s) and the source, provide a link to the Creative Commons licence, and indicate if changes were made. The images or other third party material in this article are included in the article's Creative Commons licence, unless indicated otherwise in a credit line to the material. If material is not included in the article's Creative Commons licence and your intended use is not permitted by statutory regulation or exceeds the permitted use, you will need to obtain permission directly from the copyright holder. To view a copy of this licence, visit http://creativecommons.org/licenses/by-nc/4.0/.

\section{References}

1. Khanna R, Sattin BD, Afif W et al. Review article: a clinician's guide for therapeutic drug monitoring of infliximab in inflammatory bowel disease. Aliment Pharmacol Ther. 2013;38:447-459.

2. Vermeire S, Dreesen E, Papamichael K, Dubinsky MC. How, when, and for whom should we perform therapeutic drug monitoring? Clin Gastroenterol Hepatol. 2020;18:1291-1299.

3. Papamichael K, Cheifetz AS. Therapeutic drug monitoring in inflammatory bowel disease: for every patient and every drug? Curr Opin Gastroenterol. 2019;35:302-310.
4. Vande Casteele N, Ferrante M, Van Assche G et al. Trough concentrations of infliximab guide dosing for patients with inflammatory bowel disease. Gastroenterology. 2015;148:1320-1329.

5. Feuerstein JD, Nguyen GC, Kupfer SS, Falck-Ytter Y, Singh S, American gastroenterological association institute guideline on therapeutic drug monitoring in inflammatory bowel disease. Gastroenterology. 2017;153:827-834.

6. Kelly OB, Donnell SO, Stempak JM, Steinhart AH, Silverberg MS. Therapeutic drug monitoring to guide infliximab dose adjustment is associated with better endoscopic outcomes than clinical decision making alone in active inflammatory bowel disease. Inflamm Bowel Dis. 2017;23:1202-1209.

7. Steenholdt $\mathrm{C}$, Brynskov J, Thomsen $\mathrm{OO}$ et al. Individualised therapy is more cost-effective than dose intensification in patients with Crohn's disease who lose response to anti-TNF treatment: a randomised, controlled trial. Gut. 2014;63:919-927.

8. Huang VW, Prosser C, Kroeker KI et al. Knowledge of fecal calprotectin and infliximab trough levels alters clinical decisionmaking for IBD outpatients on maintenance infliximab therapy. Inflamm Bowel Dis. 2015;21:1359-1367.

9. Papamichael K, Chachu KA, Vajravelu RK et al. Improved long-term outcomes of patients with inflammatory bowel disease receiving proactive compared with reactive monitoring of serum concentrations of infliximab. Clin Gastroenterol Hepatol. 2017;15:1580-1588.

10. Papamichael K, Juncadella A, Wong D et al. Proactive therapeutic drug monitoring of adalimumab is associated with better longterm outcomes compared with standard of care in patients with inflammatory bowel disease. J Crohns Colitis. 2019;13:976-981.

11. Sandborn WJ, Gasink C, Gao LL et al. Ustekinumab induction and maintenance therapy in refractory Crohn's disease. $N$ Engl $J$ Med. 2012;367:1519-1528.

12. Sands BE, Sandborn WJ, Panaccione R et al. Ustekinumab as induction and maintenance therapy for ulcerative colitis. $N \mathrm{Engl}$ J Med. 2019;381:1201-1214.

13. Adedokun OJ, $\mathrm{Xu} \mathrm{Z}$, Gasink $\mathrm{C}$ et al. Pharmacokinetics and exposure response relationships of ustekinumab in patients with Crohn's disease. Gastroenterology. 2018;154:1660-1671.

14. Battat R, Kopylov U, Bessissow T et al. Association between ustekinumab trough concentrations and clinical, biomarker, and endoscopic outcomes in patients with Crohn's disease. Clin Gastroenterol Hepatol. 2017;15:1427-1434.

15. Painchart $\mathrm{C}$, Brabant $\mathrm{S}$, Duveau $\mathrm{N}$ et al. Ustekinumab serum trough levels may identify suboptimal responders to ustekinumab in Crohn's disease. Dig Dis Sci. 2020;65:1445-1452.

16. Rowan CR, Keegan D, Byrne $\mathrm{K}$ et al. Subcutaneous rather than intravenous ustekinumab induction is associated with comparable circulating drug levels and early clinical response: a pilot study. Aliment Pharmacol Ther. 2018;48:333-339.

17. Verstockt B, Dreesen E, Noman M et al. Ustekinumab exposureoutcome analysis in Crohn's disease only in part explains limited endoscopic remission rates. J Crohns Colitis. 2019;13:864-872.

18. Marini C, Gils A, Shankar G et al. Comparison of the KU Leuven ustekinumab concentration assay and the antibodies-to-ustekinumab assay with assays developed at Janssen R\&D and used in clinical studies of IBD patients. J Crohns Colitis. 2018;12:S439.

19. Verdon C, Vande Casteele N, Heron V et al. Comparison of serum concentrations of ustekinumab obtained by three commercial assays in patients with Crohns disease. Gastroenterology. 2019;156:S1141.

20. van Schouwenburg PA, Bartelds GM, Hart MH, Aarden L, Wolbink GJ, Wouters D. A novel method for the detection of antibodies to adalimumab in the presence of drug reveals "hidden" immunogenicity in rheumatoid arthritis patients. J Immunol Methods. 2010;362:82-88. 
21. Menting SP, Coussens E, Pouw MF et al. Developing a therapeutic range of adalimumab serum concentrations in management of psoriasis: a step toward personalized treatment. JAMA Dermatol. 2015;151:616-622.

22. Afif W, Loftus EV Jr, Faubion WA et al. Clinical utility of measuring infliximab and human anti-chimeric antibody concentrations in patients with inflammatory bowel disease. Am J Gastroenterol. 2010;105:1133-1139.

23. Tracey D, Klareskog L, Sasso EH, Salfeld JG, Tak PP. Tumor necrosis factor antagonist mechanisms of action: a comprehensive review. Pharmacol Ther. 2008;117:244-279.

24. D'Haens $G$, Vermeire $S$, Lambrecht $G$ et al. Increasing infliximab dose based on symptoms, biomarkers, and serum drug concentrations does not increase clinical, endoscopic, and corticosteroidfree remission in patients with active luminal Crohn's disease. Gastroenterology. 2018;154:1343-1351.

25. Waljee AK, Wallace BI, Cohen-Mekelburg S et al. Development and validation of machine learning models in prediction of remission in patients with moderate to severe Crohn disease. JAMA Netw Open. 2019;2:e193721.
26. Bressler B, Panaccione R, Fedorak RN, Seidman EG. Clinicians' guide to the use of fecal calprotectin to identify and monitor disease activity in inflammatory bowel disease. Can J Gastroenterol Hepatol. 2015;29:369-372.

27. Rosenfeld G, Greenup AJ, Round A et al. FOCUS: Future of fecal calprotectin utility study in inflammatory bowel disease. World $J$ Gastroenterol. 2016;22:8211-8218.

28. Dreesen E, Baert F, Laharie D et al. Monitoring a combination of calprotectin and infliximab identifies patients with mucosal healing of Crohn's disease. Clin Gastroenterol Hepatol. 2020;18:637-646.

29. Feagan BG, Sandborn WJ, Gasink C et al. Ustekinumab as induction and maintenance therapy for Crohn's disease. $N$ Engl J Med. 2016;375:1946-1960.

Publisher's Note Springer Nature remains neutral with regard to jurisdictional claims in published maps and institutional affiliations. 